\title{
One-Electron Equations for Embedded Electron Density and Their Applications to Study Electronic Structure of Atoms and Molecules in Condensed Phase
}

\author{
Marcin Dulak, Ruslan Kevorkyants, Fabien Tran, and Tomasz A. Wesolowski*
}

\begin{abstract}
Recent applications of one-electron equations for embedded electron density introduced originally for multi-level modeling of solvated molecules (T.A. Wesolowski, A. Warshel, J. Phys. Chem. 1993, 97, 8050) are reviewed. The considered applications concern properties directly related to the electronic structure of molecules (or an atom) in condensed phase such as: i) localized electronic excitations in a chromophore involved in a hydrogen-bonded intermolecular complex; ii) UV/Nis spectra of acetone in water; and iii) energy levels of $f$-orbitals for lanthanide cations in a crystalline environment. For each case studied, the embedding potential is represented graphically and its qualitative features are discussed.
\end{abstract}

Keywords: Density functional theory $\cdot f$-Levels of lanthanides · Multi-Level computer simulations . Orbital-free embedding $\cdot$ Solvatochromism

\section{Introduction}

Computer modeling of a molecule or other polyatomic system in condensed phase, e.g. a solvated molecule, relies on two types of simplifications: i) the simplification of the model of the real system and ii) the simplification of the method to solve approximately the Schrödinger equation, which is the basic physical law governing the behavior of polyatomic systems. As far as the method is concerned, the most accurate quantum chemistry methods are, unfortunately, applicable only for systems comprising a small number of atoms. The methods for solving

${ }^{*}$ Correspondence: Dr. T.A. Wesolowski Department of Physical Chemistry University of Geneva

30, quai Ernest-Ansermet

$\mathrm{CH}-1211$ Geneva 4

Tel.: +41223796101

Fax: +412237965 18

E-Mail: tomasz.wesolowski@chiphy.unige.ch

http://lcta.unige.ch/ tomek/ numerically the Schrödinger equation (even very approximately) are not applicable for systems of the nano- and macroscopic size. Therefore, a computer model can represent only a fragment of the real macroscopic system. A brute-force strategy in modeling such systems consists of using a model comprising the molecule(s) of primary interest and as large as possible number of atoms in its(their) environment. Such a strategy limits the accuracy of the obtained results because only methods providing approximate solutions of the Schrödinger equation of medium quality can be applied in practice for systems including several hundreds of atoms. An alternative to finding approximate solutions of the Schrödinger equation for the whole system is to use the embedding strategy. The main idea of this strategy is to use different approximate methods to solve the Schrödinger equation for different parts of the whole system. In particular, not to solve the Schrödinger equation for the environment at all, but to take into account its presence by means of an additional term in the potential (embedding potential). The embedding strategy is used commonly in computer modeling of biomolecules [1], solvated molecules [2], and systems relevant for materials science [3]. The simplest practical realization of the embedding strategy, commonly applied in so-called hybrid quantum/classical methods, uses only electrostatic terms in the embedding potential, whereas the non-electrostatic effects are taken into account by means of special terms in the conformational energy. Such terms, representing exchange-repulsion or dispersion interactions for instance, are typically system-dependent.

In 1993, we have shown [4] that the embedding potential can be expressed in a system-independent way using universal functionals of two electron densities: that of the embedded system density $\left(\rho_{A}\right)$ and that of its environment $\left(\rho_{B}\right)$. It is noteworthy that electron density is well defined in both macroscopic and microscopic worlds. Therefore, the electron density partitioning strategy offers the formal framework for multi-level computer simulations where the electron densities of different parts of a real macroscopic system are described using physical laws corresponding to the size of each part. In particular, one can restrict the Kohn-Sham-like description, which uses one-electron orbitals to express the total energy of a given electron density $\left(\rho_{A}\right)$, to a subsystem of a microscopic size which is of the primary interest (a chromophore for instance). In such a case, the one-electron functions (embedded orbitals) can be obtained from Kohn-Sham like one-electron equations [4]. The electron density $\left(\rho_{B}\right)$ of the remaining part of the total system (environment), which can be of the microscopic size, is used in these equations to express the embedding component of the effective 
potential by means of universal functionals of two electron densities $\left(\rho_{A}\right.$ and $\left.\rho_{B}\right)$ in these equations. Until recently, we have been applying this embedding potential only in studies of ground-state properties, especially to determine parameters of the potential energy surfaces. Such applications have been already reviewed in this journal [5]. This type of studies have been continued in our group [6][7]. The present review concerns a new type of applications dealing with properties directly related to the electronic structure such as orbitallevels and excitation energies.

\section{The Orbital-free Embedding Formalism}

Ground-state electron density of an embedded molecule in a given environment can be derived from one-electron equations [4]:

$$
\begin{aligned}
& {\left[-\frac{1}{2} \nabla^{2}+V_{e f f}^{K S C E D}\left[\rho_{A}, \rho_{B}\right](\vec{r})\right]} \\
& \phi_{i}^{K S C E D}(\vec{r})=\varepsilon_{i}^{K S C E D} \phi_{i}^{K S C E D}(\vec{r})
\end{aligned}
$$

where: $\rho_{A}$ is the electron density of the embedded system constructed using one-electron functions $\left(\rho_{A}=2 \sum_{i=1}^{N} \phi_{i}^{K S C E D^{*}}(\vec{r}) \phi_{i}^{K S C E D}(\vec{r})\right)$ and $\rho_{B}$ is the electron density of the environment. Atomic units are applied in all equations throughout the text. The superscript KSCED (Kohn-Sham Equations with Constrained Electron Density) is used to indicate that such quantities as the effective potential, orbitals, and orbital energies, differ from the corresponding quantities in the commonly used Kohn-Sham [8] equations:

$$
\left[-\frac{1}{2} \nabla^{2}+V_{\text {eff }}^{K S}[\rho](\vec{r})\right] \phi_{i}^{K S}(\vec{r})=\varepsilon_{i}^{K S} \phi_{i}^{K S}(\vec{r})
$$

where $\rho=2 \sum_{i=1}^{N} \phi_{i}^{K S^{*}}(\vec{r}) \phi_{i}^{K S}(\vec{r})$.

The effective potential in Eqn. (1) differs from that in Eqn. (2) by additional terms describing the interactions between the two subsystems. These terms have a universal system-independent form [4] reading:

$$
\begin{aligned}
& V_{e f f}^{e m b(K S C E D)}\left[\rho_{A}, \rho_{B}\right](\vec{r})= \\
& =-\sum_{\alpha}^{N_{B}} \frac{Z_{\alpha}^{B}}{\left|\vec{R}_{\alpha}^{B}-\vec{r}\right|}+\int \frac{\rho_{B}\left(\vec{r}^{\prime}\right)}{\left|\vec{r}-\vec{r}^{\prime}\right|} d r^{\prime}+\left.\frac{\delta E_{x c}[\rho]}{\delta \rho}\right|_{\rho=\rho_{A}+\rho_{B}} \\
& -\left.\frac{\delta E_{x c}[\rho]}{\delta \rho}\right|_{\rho=\rho_{A}}+-\left.\frac{\delta T_{s}^{n a d}\left[\rho_{1}, \rho_{2}\right]}{\delta \rho_{1}}\right|_{\rho_{1}=\rho_{A}}
\end{aligned}
$$

where $N_{B}$ is the number of nuclei in subsystem $B$ and $Z_{\alpha}{ }^{B}$ is the nuclear charge of nucleus $\alpha$ in subsystem $B$. The exchange- correlation functional $E_{x c}[\rho]$ is defined as in the Kohn-Sham formalism [8] and the non-additive kinetic energy $T_{s}{ }^{n a d}\left[\rho_{1}, \rho_{2}\right]$, a functional depending on two electron densities, is defined as:

$$
T_{s}^{n a d}\left[\rho_{1}, \rho_{2}\right]=T_{s}\left[\rho_{1}+\rho_{2}\right]-T_{s}\left[\rho_{1}\right]-T_{s}\left[\rho_{2}\right]
$$

where $T_{s}[\rho]$ is the kinetic energy of the reference system of the non-interacting electrons.

Eqns. (1) and (2) have different solutions, which depend on the environment represented in the KSCED embedding potential by means of positions and electric charges of atomic nuclei and the electron density of the environment. Therefore, calculation of the changes in the electronic structure follows a simple strategy - the shift of a calculable observable is obtained as the difference between the values calculated for the two sets of one-electron functions derived from Eqn. (1) and (2), respectively. Each time the geometry of the environment changes in the process under investigation $\rho_{B}$ changes also and Eqn. (2) has to be solved again.

Practical applications of the outlined strategy involve, however, several challenging issues: i) availability of good approximations to the density functionals of unknown analytic form (all but the first two terms in the right-hand-side of Eqn. (3)), ii) an efficient computer implementation of Eqns. (1)-(3) enabling fast evaluation of embedded orbitals and observables of interest, iii) a computationally efficient way to obtain the electron density of the environment $\left(\rho_{B}\right)$. As far as the first issue is concerned, we developed a very accurate system-independent approximation to the kinetic-energy-dependent component of the embedding potential applicable for such cases where the $\rho_{A}, \rho_{B}$ overlap is small [9]. This approximation, labeled GGA97 here, uses generalized gradient approximation to $T_{s}[\rho]$ in Eqn. (4) in which the gradientdependency has the same analytical form as that of PW91 exchange functional [10] with coefficients adopted for the kinetic energy by Lembarki and Chermette [11] Therefore, applications of the orbital-free embedding formalism are currently limited to the small $\rho_{A}, \rho_{B}$ overlap cases. The absence of covalent bonding between subsystems $A$ and $B$ can be used as a practical rule of thumb for applicability of these approximations.

The second issue is of a rather technical nature but it represents a significant human effort. Recently, two efficient computer implementations of the orbital-free embedding formalism have been developed in our group, each based on codes solving Kohn-Sham equations (Eqn. (2)). The first one [12] is based on the computer code deMon2K [13] and the other one [14] is based on the code ADF [15]. Each of these codes has its strengths and weaknesses as far as implementation and applications of the orbital-free embedding formalism are concerned. Such issues will, however, not be discussed in detail here.

The third issue - generating the electron density of the environment - depends on the system under investigation. Two extreme cases can be distinguished. A hydrated organic chromophore represents the first one. In this case, the embedded subsystem is nonpolar but the geometry of the environment is flexible. Since the electronic properties of the embedded system depend on the geometry of the environment, statistical averaging using an ensemble of conformations of the environment at a given temperature is indispensable if the calculated properties are to be compared to experimental measurements. In this case, a rapid generation of $\rho_{B}$ is crucial. Two factors combine in the final quality of the results: i) the adequacy of the sample of the geometries used for averaging and ii) the quality of $\rho_{B}$ for each geometry of the environment. The effects of these two factors on the quality of the calculated average electronic-structure-dependent observables are difficult to separate. A charged or polar embedded molecule surrounded by a structurally rigid environment such as the interior or surface of a solid represents the second extreme case. Taking properly into account electronic polarization of the environment determines the overall quality of the calculated properties.

In the subsequent section the recent applications of Eqn. (1) will be reviewed. The details of calculations are provided in the original publications. In this work, such quantities as embedding potential and embedded orbitals are shown graphically to supplement the original data. The potentials, calculated using the deMon $2 \mathrm{~K}$ implementation at the local-density-approximation level, are plotted using the OpenDX [16] software. The orbitals are plotted using the Molekel 4.0 code [17].

\section{Applications}

\section{Chromophores in Condensed Phase}

Recently, we generalized the orbitalfree embedding formalism to study excited states [18]. To this end, the general framework of linear-response time-dependent density functional theory (LR-TDDFT) was used. This unified formalism is especially suited for studies of localized electronic excitations in condensed phase. Its first application concerned the effect of hydrogen bonding on the lowest excitation energies in guanine-cytosine and adenine-thymine base pairs [14]. These studies showed that 
the effect of the environment linked to the chromophore via hydrogen bonds can be accurately represented by the embedding potential of the Eqn. (3) form. Fig. 1 shows that deviations between supermolecule and embedding results do not exceed $0.05 \mathrm{eV}$ in the whole range of calculated shifts varying between -0.2 and $+0.7 \mathrm{eV}$. The effect of hydrogen bonding is not uniform for all considered low lying localized excitations. Fig. 2 shows that the embedding potential $V_{\text {eff }}^{\text {emb(KSCED) }}$ for $\rho_{A}$ and $\rho_{B}$ being the electron densities of guanine and cytosine, respectively. Indeed, $V_{\text {eff }}^{\text {emb }(K S C E D)}$ is not uniform in the different parts of the guanine molecule. A more detailed analysis of the effect of different components of $V_{\text {eff }}^{\text {emb KSCED) }}$ reveals that both its electrostatic and non-electrostatic components cannot be neglected. Fig. 2 can be used as an illustration of a very important property of $V_{e f f}^{e m b(K S C E D)}$. It depends not only on the electron density of the environment $\left(\rho_{B}\right)$ but also on that of the embedded molecule. Everywhere where the electron density $\left(\rho_{A}\right)$ is small, $V_{\text {eff }}^{\text {emb KSCED })}$ is just the sum of the electrostatic potential generated by the nuclei and electron density of subsystem $B$ and a non-electrostatic part. Its non-electrostatic component calculated by means of the approximations considered so far has been found to be strongly repulsive at large $\rho_{A}, \rho_{B}$ overlaps. Its global shape reflects, therefore, the shape of the cytosine molecule. In the space occupied by guanine, however, its global shape reveals more local features. For instance, the embedding potential changes its sign in the middle of the $\mathrm{C}=\mathrm{O}$ bond which causes its different effects on the relevant $n, \pi$, and $\pi^{*}$ orbitals localized there.
The fact that the effect of hydrogen bonding on localized electronic excitations can be adequately represented by the KSCED embedding potential using the GGA97 set of system-independent approximations brings important practical benefits as far as calculation of solvatochromic shifts is concerned. The equivalence of the LR-TDDFT excitation energies derived using either Kohn-Sham orbitals for the whole system or the embedded KSCED orbitals for the chromophore provides the basis for significant computational savings in computer simulations of solvatochromic shifts. Owing to electron density partitioning in the KSCED strategy, the ground-state level of description is used for the whole system but the excited state analysis (the most time consuming part of the calculations) is restricted to the selected subsystem only. In our recent computer modeling studies of excitation spectra of acetone solvated in water [19], large clusters (up to 250) of water molecules were used to represent the environment of this chromophore. For clusters of this size, supermolecule Kohn-Sham LR-TDDFT calculations are currently not possible. Using $V_{\text {eff }}^{e m b(K C E D)}$ incorporated into the general framework of LR-TDDFT does not involve any practical difficulties for such clusters. It was determined that water molecules beyond the radius of $8 \AA$ negligibly influence the energies of $n \rightarrow \pi^{*}$ excitations. It is important to underline that the assignment of excitations is not trivial in LR-TDDFT calculations because all pairs of occupied and unoccupied orbitals can contribute to a given excitation. In the acetone case, however, a single pair corresponding to HOMO $(n)$ and LUMO $\left(\pi^{*}\right)$ provides the dominant contribution. A sample of solvent geometries including about 50 water molecules was used in statistical averaging of excitations. This sample, corresponding to the canonical ensemble at $300 \mathrm{~K}$, and was obtained from Car-Parrinello molecular dynamics simulations. Interactions with the solvent result in an average shift of 0.23 $\pm 0.03 \mathrm{eV}$. The error bar on the calculated shift is due to a rather small number (220) of geometries in the sample. The calculated shift compares very well with the experimental value of $0.20 \pm 0.01 \mathrm{eV}$. Below, we analyze in detail the embedding potential and embedded orbitals for acetone inside a cluster of water molecules at one of the geometries from the sample used for averaging [19]. The interactions with the solvent decrease the orbital energy of HOMO by $0.54 \mathrm{eV}$ and that of LUMO by $0.29 \mathrm{eV}$ for this geometry. The interactions do not affect HOMO and LUMO in the same way which leads to a shift in the HOMO-LUMO gap amounting to $+0.25 \mathrm{eV}$. The largest change of both HOMO and LUMO orbitals occurs on the carbonyl bond. For LUMO, they are localized mostly in the plane perpendicular to that of the carbon atoms whereas they are localized in the plane of carbon atoms for HOMO. A significant effect of the solvent on the orbital energies in solvated acetone arises from the strong electric field generated by water molecules. Fig. 3 shows the change of the orbitals due to the interactions with the solvent. The energy and the shape of HOMO and LUMO are, however, not affected in the same way. Fig. 4 shows that the embedding potential generated by the electron density of the solvent molecules is repulsive in the vicinity of the carbonyl

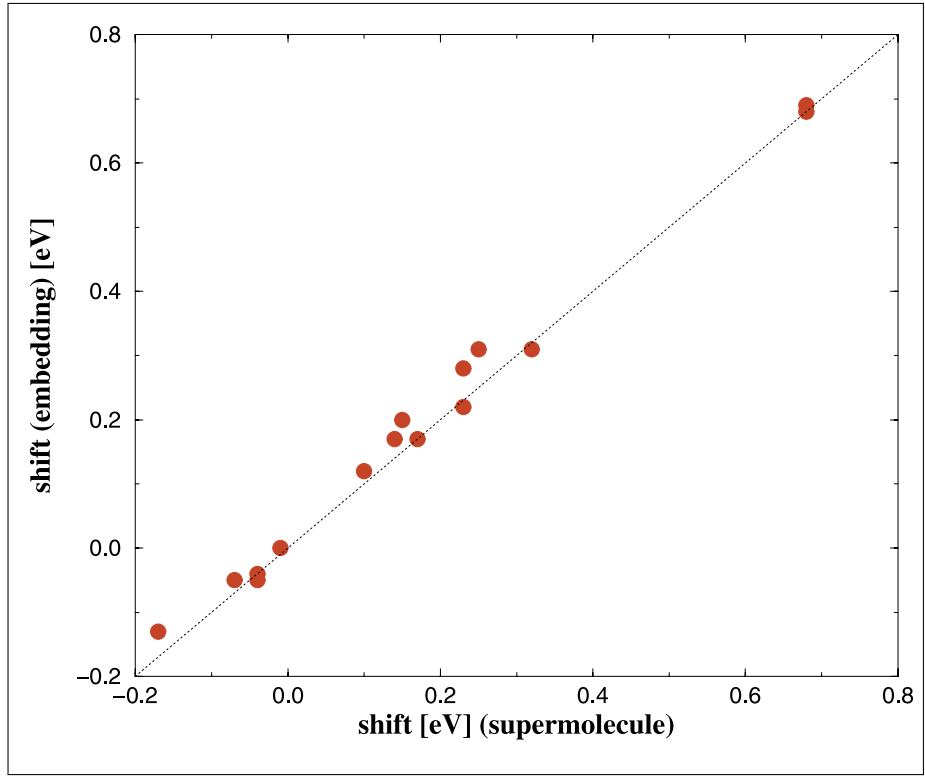

Fig. 1. The shifts of the lowest lying excitation energies localized in either guanine or cytosine in the dimer. The values calculated using embedded orbitals are plotted against reference data from supermolecule Kohn-Sham calculations.

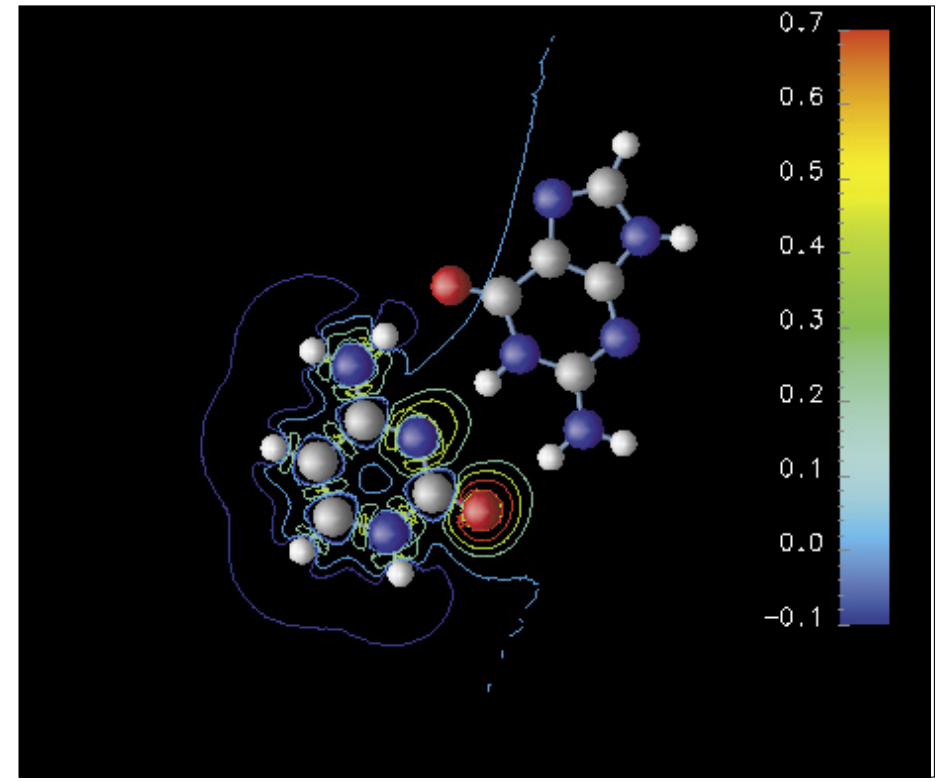

Fig. 2. Embedding potential generated by the frozen electron density of cytosine in the guanine-cytosine complex. The lines are drawn in the plane of the molecules at $0.7,0.04,0.02,0.0$, and -0.01 atomic units. 

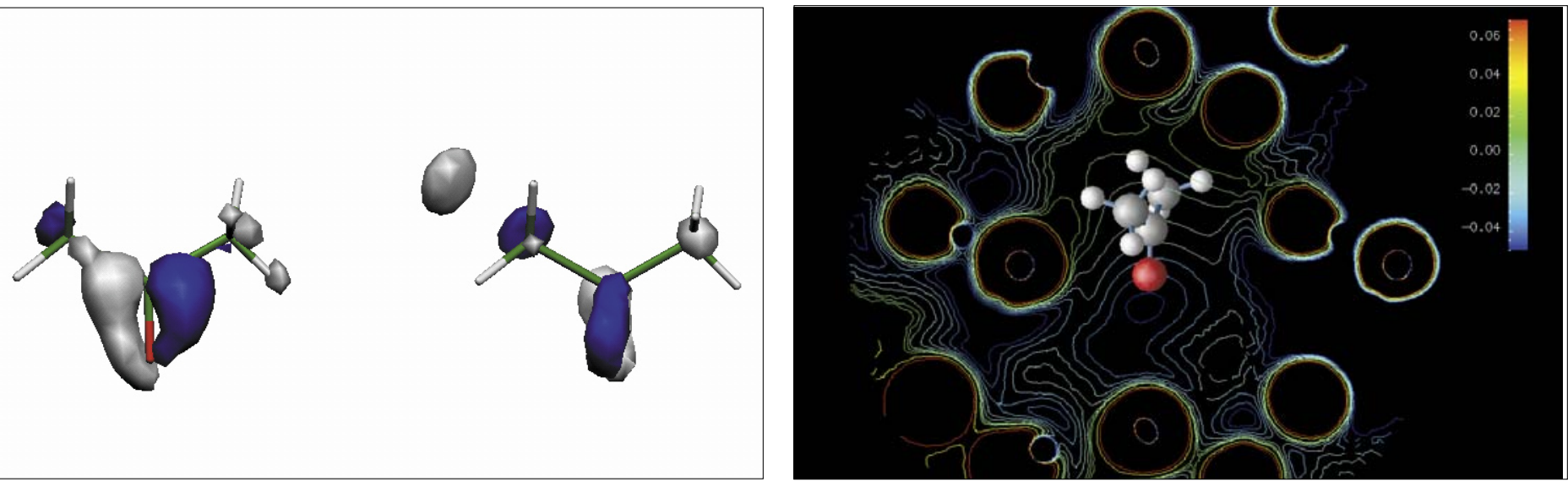

Fig. 3. Solvatation induced changes of HOMO (left) and LUMO (right) of acetone. Inside the lobes, the magnitude of the change exceeds 0.0035 atomic units.

Fig. 4. Embedding potential generated by the frozen electron density of the solvent (water) molecules. The lines are drawn at $0.07,0.04,0.01,0.005$, $0.0,-0.01,-0.02,-0.03,-0.04$, and -0.05 atomic units.

group. It is the most repulsive close to oxygen.

A smaller effect can be expected if the analyzed molecule interacts less strongly with its environment as for example in the case of dimers formed by nitrogen-containing polycyclic aromatic hydrocarbons. These molecules are targets for organic synthesis in the lab of Prof. T. Jenny (University of Fribourg) and are considered as potential building blocks of organic conducting nanocolumns. The most favorable structure of such dimers has been found to be that of slipped parallel arrangement [6]. Fig. 5 shows the dimerization-induced changes of HOMO and LUMO orbitals of $\mathrm{C}_{30} \mathrm{H}_{15} \mathrm{~N}$. It is worthwhile to notice that no apparent changes of either HOMO or LUMO occur in the vicinity of the nitrogen atom and that the changes of the orbitals are delocalized throughout the whole molecule. LUMO appears to be significantly more affected by dimerization than HOMO. Comparison of the orbital energies in the free molecule and in the dimer shows that the energies of both HOMO and LUMO are shifted towards higher values by about $0.15-0.20 \mathrm{eV}$ as the result of dimerization.

\section{Lanthanides in Crystalline \\ Environment}

Calculations of the splitting energies of $f$-levels in lanthanide centers in the crysta environment represent a challenge for firstprinciples based methods. If the lanthanide cation and its ligands are described at the Kohn-Sham level, the splitting energies are usually qualitatively wrong [20]. This was attributed to unphysical mixing of ligand orbitals with the $f$-orbitals of the lanthanide. This too strong mixing originates from the flaws of the known approximations to the exchange-correlation potential. In octahe-

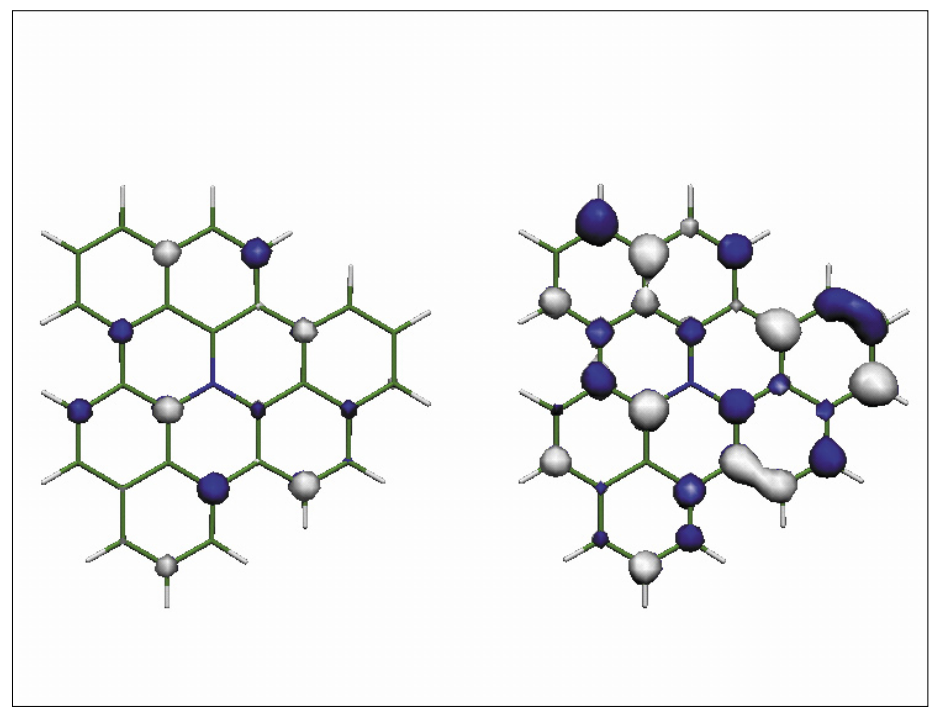

ral environment, the $f$-levels are split into three ligand-field induced levels: $\mathbf{t}_{\mathbf{2} \mathbf{u}}, \mathbf{t}_{\mathbf{1} \mathbf{u}}$, and $\mathbf{a}_{\mathbf{2}}$. Application of the orbital-free embedding potential (Eqn. (3)) to calculate the splitting energies for the whole lanthanide series shows in chloroelpasolite crystals a remarkably good agreement with experiment [21]. The calculated $\mathbf{t}_{\mathbf{2} \mathbf{u}}-\mathbf{a}_{\mathbf{2}}$ splitting energies agree typically within $10 \%$ with experiments whereas the $\mathbf{t}_{\mathbf{1} \mathbf{u}}-\mathbf{a}_{\mathbf{2} \mathbf{u}}$ ones are underestimated by about $20 \%$. For comparison, Kohn-Sham calculations lead to qualitatively wrong results [20]. It has to be underlined that the approximate functionals used in these calculations were developed based on first-principles and no experimental data concerning rare-earth elements was used. Fig. 6 shows the embedding potential $V_{e f f}^{e m b(K S C E D)}$ inside the cage formed by six $\mathrm{Cl}^{-}$anions calculated for a trial spherically symmetric electron density $\rho_{A}$. It increases the fastest along the $x, y$, or $z$ axes. Therefore, the orbitals oriented along these axes can be expected to be the least stabilized by the ligands. Indeed, the highest energy corresponds to the $\mathbf{t}_{1 \mathbf{u}}$ orbitals which have $\sigma$ character and are oriented along the $C_{4}$ axes. The $\mathbf{a}_{\mathbf{2}}$ orbital is the most compact and its lobes are oriented along the direction of the slowest increase of $V_{e f f}^{e m b(K S C E D)}$. As a result, the corresponding energy levels are the lowest. The numerical value of the splitting energies derived from our calculations can be attributed to the non-additive kinetic energy component of $\mathbf{a}_{\mathbf{2} \mathbf{u}}$. Polarization of the ligands by the cation provides also a noticeable contribution. The electrostatic contribution alone leads, however, to a qualitatively wrong picture [21].

\section{Conclusions}

Fig. 5. Dimerization-induced changes of HOMO (left) and LUMO (right) in $\mathrm{C}_{30} \mathrm{H}_{15} \mathrm{~N}$. Inside the lobes, the magnitude of the change exceeds 0.0015 atomic units.

Owing to recent improvements of the computer implementation of the orbital- 


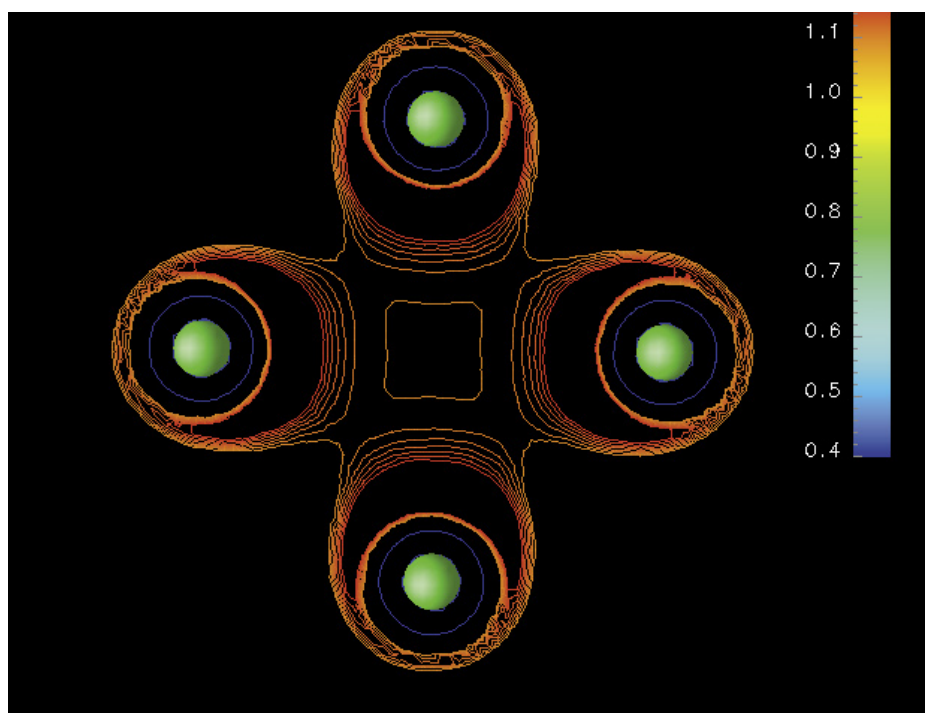

Fig. 6. Embedding potential generated by the frozen electron density of the ligands shown the plane formed by four chlorine atoms. The lines are drawn at: $1.15,1.14,1.13,1.12,1.11,1.10,1.09$, and 0.4 atomic units.

free embedding formalism, a new class of applications concerning the electronic structure of embedded molecules became recently possible. Details of these applications have been reported elsewhere [14][19][21]. Here, we have provided a general review supplemented by additional analyses involving graphical representations of the embedding potential in each discussed case. These analyses show clearly that the embedding potential given in Eqn. (3) affects the orbitals of the embedded subsystem in a highly non-uniform way. This potential comprises the $\rho_{A}$-independent electrostatic component and the remaining $\rho_{A}$-dependent part which becomes strongly repulsive at large $\rho_{A}, \rho_{B}$ overlaps. Finally, it is worthwhile to underline that the used gradientdependent approximations to the relevant components of the orbital-free embedding potential of Eqn. (3) have previously been tested and used only for deriving properties of the ground-state potential energy surface for various embedded systems. The recent new applications indicate clearly that the same approximations can be also used to study electronic structure and the excited states in particular.

\section{Acknowledgements}

Collaboration with Prof. M. Atanasov (Bulgarian Academy of Sciences, Sofia), Prof. E.-J. Baerends and Dr. J. Neugebauer (Vrije Universitait Amsterdam), Prof. M.E. Casida (Université Joseph Fourier, Grenoble), Prof. C.A. Daul and Mr. M. Zbiri (Université de Fribourg) in various parts of the reviewed research is greatly acknowledged. This research was supported by grants from Swiss National Science Foundation and Swiss Federal Office of Science and Education (D26 of COST).

Received: May 19, 2005

[1] J. Åqvist, A. Warshel, Chem. Rev. 1993, 93, 2523

[2] J. Gao, in 'Reviews in Computational Chemistry', vol. 7, Eds. K.B. Lipkowitz, D.B. Boyd, VCH, New York, 1995, p. 119.

[3] J. Sauer, P. Ugliengo, E. Garrone, V.R. Saunders, Chem. Rev. 1994, 94, 2095.

[4] T.A. Wesolowski, A. Warshel, J. Phys. Chem. 1993, 97, 8050.

[5] T.A. Wesolowski, Chimia 2002, 56, 707.

[6] F. Tran, B. Alameddine, T.A. Jenny, T.A. Wesolowski, J. Phys. Chem. A 2004, 108, 9155.

[7] F. Tran, T.A. Wesolowski, Int. J. Quantum Chem. 2005, 101, 854.
[8] W. Kohn, L.J. Sham, Phys. Rev. 1965, 140 A1133.

[9] T.A. Wesolowski, J. Chem. Phys. 1997, 106, 8516

[10] J.P. Perdew, in 'Electronic Structure of Solids'91', Eds. P. Ziesche, H. Eschrig, Akademie Verlag, Berlin, 1991, p. 11

[11] A. Lembarki, H. Chermette, Phys. Rev. A 1994, 50, 5328.

[12] M. Dulak, T.A. Wesolowski, Int. J. Quant. Chem. 2005, 101, 543.

[13] A.M. Köster, R. Flores-Moreno, G. Geudtner, A. Goursot, T. Heine, J.U. Reveles, A. Vela, D.R. Salahub, deMon, 2003, NRC, Canada.

[14] T.A. Wesolowski, J. Am. Chem. Soc. 2004, $126,11444$.

[15] ADF 2003.01, SCM, Theoretical Chemistry, Vrije Universiteit, Amsterdam, The Netherlands. http://www.scm.com

[16] OpenDX: The Open Source Software Project Based on IBM's Visualization Data Explorer, http://www.opendx.org

[17] MOLEKEL 4.0, P. Flükiger, H.P. Lüthi, S Portmann, J. Weber, Swiss Center for Scientific Computing, Manno (Switzerland), 2000.

[18] M.E. Casida, T.A. Wesolowski, Int. J. Quantum Chem. 2004, 96, 577.

[19] J. Neugebauer, M.J. Louwerse, E.J. Baerends, T.A. Wesolowski, J. Chem. Phys. 2005, 122, 09411.

[20] M. Atanasov, C.A. Daul, H.-U. Güdel, in 'Computational Chemistry: Reviews of Current Trends', Vol. 9, Ed. J. Leszczynski, World Scientific Publishing Company, Singapore, 2004.

[21] M. Zbiri, M. Atanasov, C. Daul, J.M. Garcia-Lastra, T.A. Wesolowski, Chem. Phys. Lett. 2004, 397, 441. 